\title{
Loss of beta cell function as fasting glucose increases in the non-diabetic range
}

\author{
I. F. Godsland ${ }^{1,2}$ • J. A. R. Jeffs ${ }^{1}$ D. G. Johnston ${ }^{1}$ \\ ${ }^{1}$ Department of Endocrinology and Metabolic Medicine, Division of Medicine, Faculty of Medicine, Imperial College London, \\ St. Mary's Hospital, Mint Wing 2nd Floor, London, UK \\ ${ }^{2}$ Wynn Institute, Division of Medicine, Imperial College London, London, UK
}

\section{Abstract}

Aims/hypothesis. Our aim was to define the level of glycaemia at which pancreatic insulin secretion, particularly first-phase insulin release, begins to decline.

Methods. Plasma glucose and insulin concentrations were measured during an IVGTT in 553 men with non-diabetic fasting plasma glucose concentrations. In 466 of the men C-peptide was also estimated. IVGTT insulin secretion in first and late phases was assessed by: (i) the circulating insulin response; (ii) population parameter deconvolution analysis of plasma C-peptide concentrations; and (iii) a combined model utilising both insulin and C-peptide concentrations. Measurements of insulin sensitivity and elimination were also derived by modelling analysis.
Results. As fasting plasma glucose (FPG) increased, IVGTT first-phase insulin secretion declined by $73 \%$, $71 \%$ and $68 \%$ for the three methods respectively. The FPG values at which this decline began, determined by change point regression, were $4.97,5.16$ and $5.42 \mathrm{mmol} / \mathrm{l}$ respectively. The sensitivity of late-phase insulin secretion to glucose declined at FPG concentrations above $6.0 \mathrm{mmol} / \mathrm{l}$. Insulin elimination, but not insulin sensitivity, varied with FPG.

Conclusions/interpretation. The range of FPG over which progressive loss of the first-phase response begins may be as low as 5.0 to $5.4 \mathrm{mmol} / \mathrm{l}$, with latephase insulin responses declining at FPG concentrations above $6.0 \mathrm{mmol} / \mathrm{l}$.

Keywords Beta cell function - Fasting plasma glucose · Insulin secretion - Insulin sensitivity · Modelling analysis
Received: 6 November 2003 / Accepted: 19 April 2004

Published online: 13 July 2004

(C) Springer-Verlag 2004

I. F. Godsland ( $)$

Department of Endocrinology and Metabolic Medicine, Division of Medicine, Faculty of Medicine, Imperial College London, St. Mary's Hospital,

Mint Wing 2nd Floor, London, W2 1PG, UK

E-mail: i.godsland@imperial.ac.uk

Tel.: +44-207-8866573, Fax: +44-207-8861790

Abbreviations: f, fractional hepatic insulin throughput . FPG, fasting plasma glucose $\cdot$ FSD, fractional standard deviation $\cdot \mathrm{k}_{\mathrm{c}}$, the plasma $\mathrm{C}$-peptide elimination constant . $\mathrm{k}_{\mathrm{i}}$, the plasma insulin elimination constant $\cdot$ MFG, mean fasting glucose $\cdot$ SG, minimal model glucose effectiveness · SI, minimal model insulin sensitivity

In the course of this work, our colleague and co-author James Jeffs died unexpectedly after a short illness. His contribution will be greatly missed.

\section{Introduction}

The respective roles of insulin deficiency and insulin resistance in the development of Type 2 diabetes mellitus remain controversial $[1,2]$. In response to a glucose stimulus, two phases of insulin secretion may be distinguished [3]. The first occurs as an immediate response to a rise in blood glucose concentrations. Low first-phase insulin release is an early abnormality in deteriorating glucose homeostasis and predicts the subsequent development of Type 2 diabetes [4, 5, 6, 7]. Late-phase insulin release is more prolonged, but of lesser amplitude. It includes the progressively increasing second phase of insulin secretion seen in response to sustained hyperglycaemia [3], and also the compensatory insulin secretion that is the feedback response to a continued increase in circulating glucose. This late-phase insulin secretion may seem to be preserved even when glucose metabolism is defective, 
but insulin levels are low relative to the prevailing blood glucose concentration [8]. Recently, it has been suggested that the substantial extraction of newly secreted insulin that takes place in the liver $[9,10,11]$ might be a factor in the control of plasma insulin concentrations and glucose homeostasis $[12,13]$.

The cut-off limit for defining diabetes - a fasting plasma glucose (FPG) concentration of $7.0 \mathrm{mmol} / \mathrm{l}-$ was selected with reference to risk of microvascular disease $[14,15]$. Impaired fasting glucose (FPG 6.1 to $<7.0 \mathrm{mmol} / \mathrm{l}$ ) was selected with reference to risks of Type 2 diabetes [16] and coronary heart disease [17]. However, a recent prospective analysis of subjects in whom FPG levels were initially below $6.1 \mathrm{mmol} / \mathrm{l}$ suggested an optimal FPG cut-off of 5.4-5.5 mmol/1 for identifying those at risk of Type 2 diabetes (in the following 5 years) [18]. This suggests that beta cell failure may begin at FPG levels conventionally considered normal, and support for this comes from previous studies on the relationships between glycaemia and insulin concentrations [8, 19, 20]. However, the FPG level at which insulin secretion begins to decline has yet to be defined precisely and the relative contributions of first-phase and late-phase insulin secretion, insulin sensitivity and insulin elimination to variation in glucose homeostasis and plasma insulin concentrations in non-diabetic glucose homeostasis have yet to be fully elucidated.

Previous studies have generally inferred pancreatic insulin secretion from insulin concentrations. Substantial and variable uptake of newly secreted insulin by the liver, a short but variable plasma half-life for insulin and variation in the distribution space for insulin may each have had a confounding effect in such studies. We have, therefore, investigated relationships between glucose homeostasis, stimulated insulin secretion and insulin elimination and sensitivity in a large group of men with FPG levels in the non-diabetic range using four different approaches for quantifying pancreatic insulin secretion.

\section{Subjects and methods}

Design. The Heart Disease and Diabetes Risk Indicators in a Screened Cohort (HDDRISC) study is an open cohort study of metabolic risk factors for the development of coronary heart disease and diabetes mellitus (described in detail elsewhere [21, 22]). The study derived from a company health programme in which, from 1971 to 2000, participants received a range of metabolic, clinical and laboratory evaluations. Participants in the programme underwent an IVGTT. The present analysis concerns the 553 white males who, between 1987 and 1997, underwent an IVGTT and who had an FPG of less than $7.0 \mathrm{mmol} / \mathrm{l}$ and were not known to have any abnormality of glucose metabolism. Full informed consent for the study was obtained in each case and local ethics committee approval was given.

Procedures. As preparation for the IVGTT, participants were instructed to consume more than $200 \mathrm{~g}$ /day of carbohydrate in their diet for 3 days before the test, to have fasted overnight (for at least $12 \mathrm{~h}$ ), and to have taken only water and refrained from cigarette smoking on the morning of the test. Height and weight were measured and a clinical history was taken. An indwelling cannula was inserted into an antecubital vein in each arm. With the volunteer semi-recumbent, blood samples were taken for fasting plasma and serum measurements. All samples were kept on ice, plasma or serum was separated within $1 \mathrm{~h}$ of being taken and routine biochemical variables were measured on the same day. Plasma samples for measurement of insulin and C-peptide were frozen immediately. An intravenous glucose injection was then given $(0.5 \mathrm{~g}$ glucose $/ \mathrm{kg}$ body weight as a $50 \% \mathrm{w} / \mathrm{v}$ solution of dextrose, given over $3 \mathrm{~min}$ ) via the cannula in the opposite arm to the sampling arm. Blood samples $(10 \mathrm{ml})$ were then taken at $3,5,7,10,15,20,30,45,60,75$, $90,120,150$ and $180 \mathrm{~min}$ to measure plasma glucose, insulin and C-peptide.

Laboratory measurements. Plasma glucose concentrations were measured on the day of sampling by a glucose oxidase procedure [23]. Plasma insulin concentrations were measured on samples stored at $-20{ }^{\circ} \mathrm{C}$ by a radioimmunoassay procedure [24]. Plasma C-peptide concentrations were measured using the radioimmunoassay kit supplied by Guildhay (Surrey, UK). This kit ceased to be available before all IVGTT plasmas had been analysed. As a consequence, C-peptide measurements were made on only 466 of the 553 IVGTT subjects. Quality control was monitored with pooled plasmas kept in frozen store, commercially available lyophilised sera and by participation in national schemes (National External Quality Assurance Scheme [NEQAS] and Radioimmunoassay Quality Assurance Scheme [RIQAS]). Particular attention was given to maintaining long-term continuity of measurement with replicate assay of previously analysed samples held in frozen store, extensive comparisons when there was any change in assay methodology and examination of long-term measurement variation for any signs of assay drift. Within- and between-batch coefficients of variation ranged between 2 to $3 \%$ (plasma glucose), 4 to $6 \%$ (plasma insulin) and 7 to $9 \%$ (plasma C-peptide).

IVGTT modelling analysis. Insulin sensitivity (SI) and glucose effectiveness (SG; glucose-dependent glucose elimination rate at basal insulin concentration) were determined using the minimal model of glucose disappearance $[25,26]$, with a model identification programme custom-written in Fortran 77 . The relatively high glucose dose $(0.5 \mathrm{~g} / \mathrm{kg})$ used by us provides for a high rate of model identification and good correlation with the reference euglycaemic clamp technique $(r=0.92)$, without recourse to augmentation of insulin concentrations by tolbutamide or insulin injection [27, 28]. These modifications were, in any case, precluded in the present study, since endogenous late-phase pancreatic insulin secretion was to be measured.

Measures derived from plasma insulin concentration provide an index of pancreatic insulin secretion, but may be confounded by hepatic extraction of newly secreted insulin and by peripheral insulin elimination. Measures of pre-hepatic pancreatic insulin secretion were derived using C-peptide concentrations. C-peptide is secreted from the pancreas simultaneously and in equimolar quantities with insulin but is not taken up by the liver and, once in the peripheral circulation, has simpler distributional characteristics [29]. This has allowed effective working simplifications of C-peptide behaviour, which enable model-based estimates of the true pancreatic insulin secretion rate to be derived. The procedures used in the present study were: 
1. Population parameter deconvolution. The volume of distribution of C-peptide and rate constants for the distribution of C-peptide in a two-compartment model are estimated using published regression equations relating these variables to age, body surface area and sex, and obesity and diabetes status [30]. These estimated parameters are then used in a deconvolution analysis in which the pancreatic insulin secretion rate profile responsible for the observed IVGTT plasma C-peptide concentration profile is calculated.

2. The combined model of pancreatic insulin secretion. This approach uses the IVGTT insulin and C-peptide profiles and exploits the fact that these derive from a common insulin secretion rate profile [9]. The analysis determines an index of fractional hepatic insulin throughput (f), the plasma insulin elimination constant $\left(\mathrm{k}_{\mathrm{i}}\right)$, the plasma C-peptide elimination constant $\left(\mathrm{k}_{\mathrm{c}}\right)$ and pancreatic insulin secretion rate per unit $\mathrm{C}$-peptide distribution volume at successive time points during the IVGTT [31]. The simplifying assumptions are made that newly secreted insulin and C-peptide enter single compartments of distribution after passing through the liver and that hepatic uptake of newly secreted insulin during the IVGTT is constant. Percentage hepatic insulin extraction of insulin can be calculated as: $\left(1-\left(\mathrm{f} \times\left(\mathrm{V}_{\mathrm{i}} / \mathrm{V}_{\mathrm{c}}\right)\right) \times 100\right.$, where $\mathrm{V}_{\mathrm{i}}$ is the volume of distribution of insulin, estimated as $7 \%$ of body weight [32] and $\mathrm{V}_{\mathrm{c}}$ is the volume of distribution of C-peptide, estimated as $12.5 \%$ of body weight [9].

3. The extended combined model of pancreatic insulin secretion. This resembles the combined model but incorporates a second compartment of C-peptide distribution [33].

For a minimal model analysis to be acceptable, parameter estimates were required to be positive and have fractional standard deviations (FSDs) of less than 100\%. For combined and extended combined model analyses to be acceptable, percent hepatic extractions and model parameters had to be positive and have FSDs of less than $500 \%$. This relaxation of the conventional upper limit of $100 \%$ was allowed after a comparison of the behaviour of model parameters from acceptable identifications (no parameter with FSD >100\%) with the behaviour of parameters from borderline identifications (any parameter with FSD 100-500\%). Significant correlations between model parameters and measures that would be expected to be physiologically related to them were equally apparent with acceptable and borderline acceptable identifications (unpublished observations). This suggests that although model parameters with FSDs of 100 to $500 \%$ were associated with wide confidence intervals, they were, nevertheless, point estimates that behaved in an equivalent manner to model parameters from fully acceptable identifications. Occasional negative secretion rates returned in the pancreatic insulin secretion analyses were taken as zero.

Data analysis. The fasting plasma glucose concentration was expressed as the mean of two fasting measurements made before starting the IVGTT (mean fasting glucose, MFG). IVGTT glucose elimination was expressed as the k value: the slope of the regression line for the natural log of the IVGTT glucose concentrations between 20 and $60 \mathrm{~min}$ (we have found that this interval provides the best fit to a monoexponential decay curve with the relatively high glucose dose we use). The net increment in IVGTT insulin area under the curve was calculated using the trapezium rule. The net increments from 0 to $10 \mathrm{~min}$ and 10 to $180 \mathrm{~min}$ were taken as measures of first-phase and late-phase IVGTT plasma insulin response respectively. Net pancreatic insulin secretion during the IVGTT was similarly calculated from the model-derived estimates of pancreatic in- sulin secretion rates at each IVGTT sampling point. Firstphase pancreatic insulin secretion was estimated up to the minimum secretion rate encountered during the first $10 \mathrm{~min}$ of the IVGTT. Late-phase secretion was then the difference between net secretion and first-phase secretion.

Statistical analyses were carried out using the STATA 6 statistical package (Stata, College Station, Tex., USA). For subsequent parametric statistical analysis, insulin sensitivity measures were square-root transformed [27]. Otherwise measures were log transformed, as appropriate, to normalise their distributions. Variation between insulin secretion, elimination and sensitivity and glucose homeostasis measures and demographic variables was explored by univariate and multiple linear regression analysis. Measures were standardised to the mean age and BMI of the groups using the regression coefficients from these analyses. Geometric means for age and BMI-standardised insulin secretion, elimination and sensitivity measures were derived within ranges of successively poorer glucose homeostasis as follows: MFG <4.75, 4.75-5.00, 5.00-5.25, 5.25-5.50, 5.50-5.75, 5.75-6.00, 6.00-6.25, 6.25-7.00 mmol/1; IVGTT k value: $>2.4,2.1-2.4,1.8-2.1,1.5-1.8,1.2-1.5,1.0-1.2$, $0.8-1.0,<0.8$ per min. Univariate correlations were explored using Pearson correlation coefficients. Prediction of fasting glucose concentrations and IVGTT $\mathrm{k}$ values was investigated by multiple linear regression analysis. Inflection points in the association between measures of insulin secretion and MFG were explored by change point regression analysis [34]. According to this technique, two linear models, predicting insulin secretion from MFG, are fitted to the data above and below a defined MFG threshold or change point, where the two regression lines meet. Variation in the residual sums of squares with variation in the value assigned to the defined threshold is explored and the threshold that achieves the minimum residual sums of squares is taken as the change point. This model is then tested against the single linear model to establish whether a significant improvement in fit has been achieved. The sensitivity of latephase pancreatic insulin secretion to the accompanying glucose stimulus was quantified as the coefficient for the regression of late-phase insulin secretion on the IVGTT incremental glucose area between 10 and $180 \mathrm{~min}$, within each range of MFG and IVGTT k value.

\section{Results}

Group characteristics. These are shown in Table 1. Of the 553 men, 13 were taking lipid-lowering agents, 14 beta blockers, 13 diuretics, 4 other blood-pressurelowering agents, and 9 uric-acid-lowering agents. Of the 553 IVGTTs, minimal model analysis was successful in 545 cases. Of the 466 IVGTTs for which C-peptide was measured, combined model results from 408 were available for statistical analysis, there being 58 outright model failures and 65 identifications associated with model parameter coefficients of variation of 100 to $500 \%$. For the extended combined model, there were 95 outright model failures and 128 identifications associated with model parameter coefficients of variation of 100 to $500 \%$. No further analysis was undertaken with results from the extended combined model.

Mean age and BMI, and age- and BMI-standardised means for SI, SG and insulin secretion and elimi- 
Table 1. Group characteristics (means and ranges, and frequencies)

\begin{tabular}{lc}
\hline Number & 553 \\
\hline Age (years) & $50.1(26-78)$ \\
Body mass index $\left(\mathrm{kg} / \mathrm{m}^{-2}\right)$ & $25.7(18.6-41.1)$ \\
Systolic blood pressure $(\mathrm{mm} \mathrm{Hg})$ & $125.7(85-200)$ \\
Diastolic blood pressure $(\mathrm{mm} \mathrm{Hg})$ & $79.1(55-120)$ \\
Current cigarette smoking $(\%)$ & \\
Never & 37.9 \\
Previous & 42.2 \\
$<25 / \mathrm{d}$ & 18.3 \\
$>25 / \mathrm{d}$ & 1.6 \\
Alcohol intake $(\%)$ & \\
Never & 2.7 \\
$<28$ units/week & \\
$>28$ units/week & \\
Physical activity $(\%)$ & 71.1 \\
Never & 26.2 \\
Non-aerobic & \\
Regular walking & 43.9 \\
Family history of diabetes $(\%)$ & 47.2 \\
None & 8.9 \\
Sibling or parent & \\
Other relative & 85.6 \\
\hline
\end{tabular}

a 1 unit of alcohol approximately to $8 \mathrm{~g}$ of absolute alcohol

nation characteristics according to MFG and IVGTT k value category are shown in Tables 2 and 3 respectively. Regression analyses in which MFG and $\mathrm{k}$ are predicted by insulin secretion, sensitivity and elimination and age and BMI, with significances for each regression coefficient, are shown in Table 4.

Associations with insulin sensitivity and glucose effectiveness. Categories of deteriorating glucose homeostasis (i.e. increasing MFG and decreasing $\mathrm{k}$ ) displayed no marked trend in mean age- and BMI-standardised SI. Categories of deteriorating glucose homeostasis displayed a decreasing mean age- and BMIstandardised SG (MFG by $25 \%$ and $\mathrm{k}$ by $65 \%$ ). In regression analysis, decreasing SG was a significant independent predictor of deteriorating glucose homeostasis (MFG and $\mathrm{k}$ both $p<0.001$ ).

Associations with insulin concentration measurements. Categories of deteriorating glucose homeostasis displayed an increasing mean age- and BMI-standardised net increment in IVGTT insulin concentrations. In regression analysis, increasing net increment in IVGTT insulin concentrations was a significant independent predictor of declining glucose homeostasis (MFG $p<0.001$ and $\mathrm{k} p<0.05$ ). Categories of deteriorating glucose homeostasis showed a marked decline in mean age- and BMI-standardised IVGTT firstphase plasma insulin (MFG to $27 \%$ and and $\mathrm{k}$ to $22 \%$ of their highest values, i.e by $73 \%$ and $78 \%$ respectively). In regression analysis, decreasing IVGTT first-phase plasma insulin was a significant independent predictor of deteriorating glucose homeostasis (MFG and $\mathrm{k}$ both $p<0.001$ ). Categories of deteriorating glucose homeostasis showed an increase in mean age- and BMI-standardised IVGTT late-phase plasma insulin (MFG by $48 \%$ and $\mathrm{k}$ by $37 \%$ ). In regression analysis, increasing IVGTT late-phase plasma insulin was a significant independent predictor of deteriorating glucose homeostasis (MFG and $\mathrm{k}$ both $p<0.001$ ). IVGTT first-phase and late-phase plasma insulin were inversely correlated $(r=0.15, p<0.001)$.

Associations with pancreatic insulin secretion (population parameter deconvolution). Categories of deteriorating glucose homeostasis showed an increase in mean age- and BMI-standardised net incremental IVGTT insulin secretion, assessed by population parameter deconvolution. In regression analysis, increasing net incremental IVGTT insulin secretion was a significant independent predictor of deteriorating glucose homeostasis (MFG $p<0.05$ and $\mathrm{k} p<0.001$ ). Categories of deteriorating glucose homeostasis showed a marked decline in mean age- and BMI-standardised IVGTT first-phase secretion (MFG to $29 \%$ and $\mathrm{k}$ to $36 \%$ of their highest values, i.e. by $71 \%$ and $64 \%$ respectively). In regression analysis, decreasing IVGTT first-phase secretion was a significant independent predictor of deteriorating glucose homeostasis (MFG and $\mathrm{k}$ both $p<0.001$ ). Categories of deteriorating glucose homeostasis indicated an increase in mean ageand BMI-standardised IVGTT late-phase secretion (MFG by $16 \%$ and $\mathrm{k}$ by $255 \%$ ). In regression analysis, increasing IVGTT late-phase secretion was a significant independent predictor of deteriorating glucose homeostasis (MFG $p<0.05$ and $\mathrm{k} p<0.001$ ). IVGTT first-phase and late-phase secretion by population parameter deconvolution showed no association $(r=0.01$, $p=\mathrm{NS})$.

Associations with pancreatic insulin secretion (combined model). Categories of deteriorating glucose homeostasis showed an increase in mean age- and BMIstandardised net incremental IVGTT insulin secretion, assessed by the combined model. In regression analysis, increasing net incremental IVGTT insulin secretion was a significant independent predictor of deteriorating glucose homeostasis (MFG and $\mathrm{k}$ both $p<0.05)$. Categories of deteriorating glucose homeostasis showed a marked decline in mean age- and BMI-standardised IVGTT first-phase secretion (MFG to $32 \%$ and $\mathrm{k}$ to $46 \%$ of their highest values, i.e. by $68 \%$ and $54 \%$ respectively). In regression analysis, declining IVGTT first-phase secretion was a predictor of deteriorating glucose homeostasis (MFG and $\mathrm{k}$ both $p<0.001)$. Categories of deteriorating glucose homeostasis showed an increase in mean age- and BMI-stan- 

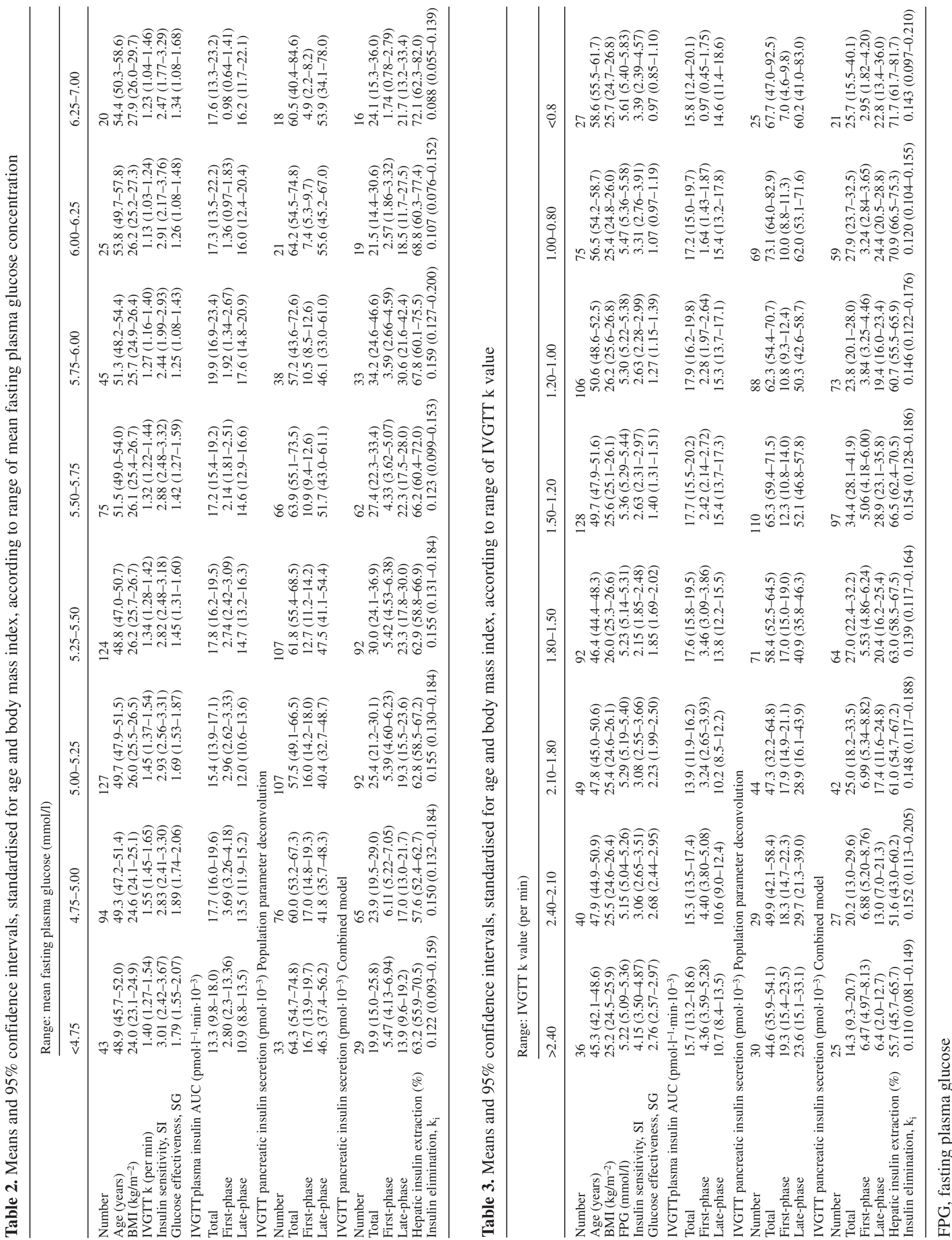
Table 4. Regression coefficients for predictors of mean fasting plasma glucose (MFG) and IVGTT k value

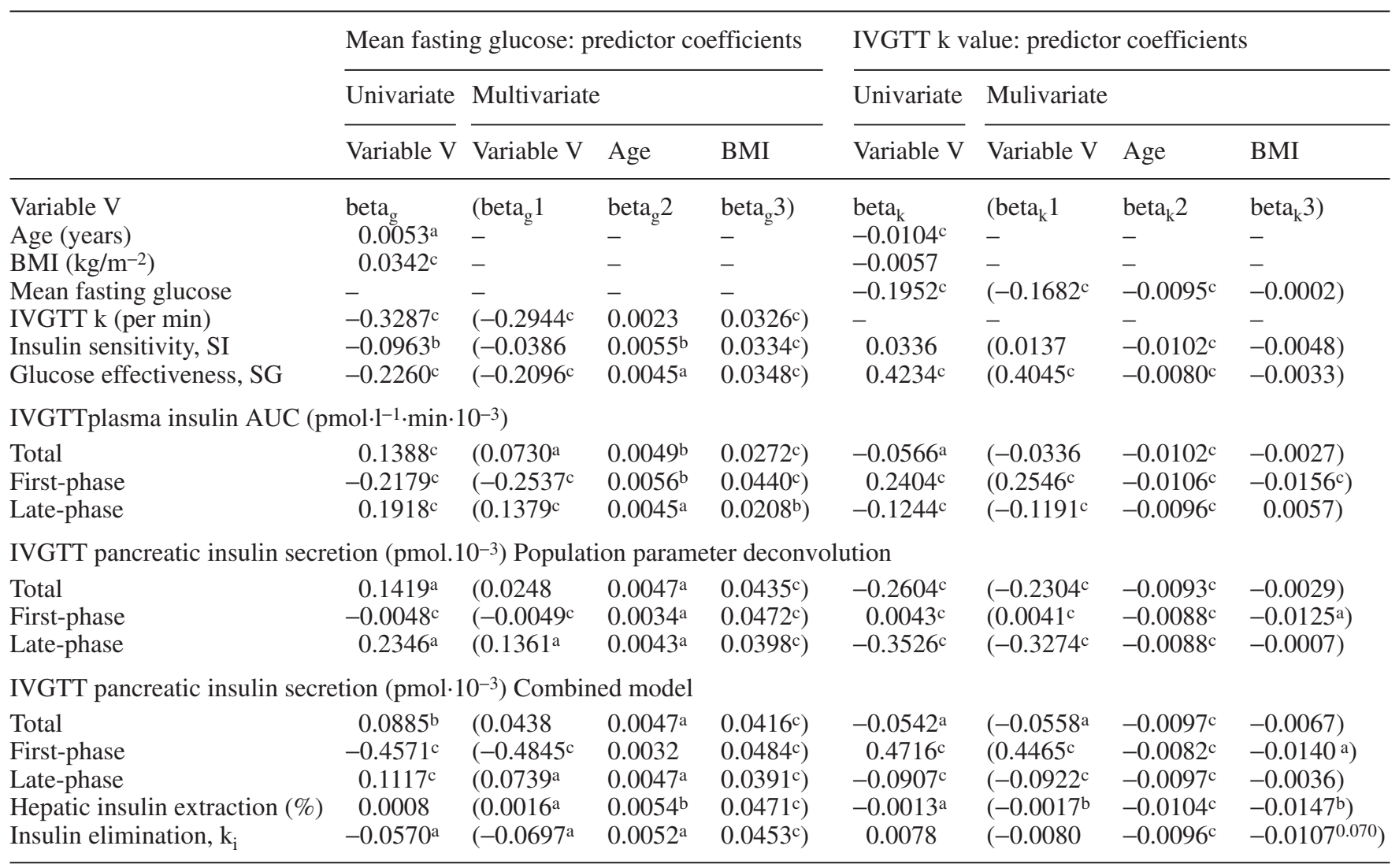

Univariate analysis derives from the following regression equations: mean fasting glucose $=$ beta $_{\mathrm{g}}{ }^{*}$ variable $\mathrm{V}+$ constant; IVGTT $\mathrm{k}$ value $=$ beta $_{\mathrm{k}}{ }^{*}$ variable $\mathrm{V}+$ constant. Multivariate analysis derives from the following equations: mean fasting glucose $=$ beta $_{\mathrm{g}} 1 *$ variable $\mathrm{V}+$ beta $_{\mathrm{g}} 2 * \mathrm{Age}+$ beta $_{\mathrm{g}} 3 * \mathrm{BMI}+$

dardised IVGTT late-phase secretion by the combined model (MFG to 53\% and $\mathrm{k}$ to $342 \%$ ). In regression analysis, increasing IVGTT late-phase secretion was a significant independent predictor of deteriorating glucose homeostasis (MFG $p<0.05$ and $\mathrm{k} p<0.001$ respectively). IVGTT first-phase and late-phase secretion by the combined model were inversely correlated $(r=0.33, p<0.001)$.

Associations with measures of insulin elimination. Categories of deteriorating glucose homeostasis showed an increase in mean age- and BMI-standardised percentage of newly secreted insulin extracted by the liver (MFG by $14 \%$ and $\mathrm{k}$ by $29 \%$ ). In regression analysis, the increasing percentage of newly secreted insulin extracted by the liver was a significant independent predictor of deteriorating glucose homeostasis (MFG $p<0.05$ and $\mathrm{k} p<0.01)$. Categories of increasing MFG showed a decrease in mean age- and BMI-standardised plasma insulin elimination rate (by $28 \%$ ), but categories of decreasing $\mathrm{k}$ showed little variation in the rate of plasma insulin elimination. In regression analysis, a decreasing plasma insulin elimi- constant; IVGTT $\mathrm{k}$ value $=$ beta $_{\mathrm{k}} 1 *$ variable $\mathrm{V}+$ beta $_{\mathrm{k}} 2 *$ Age + beta $_{\mathrm{k}} 3 * \mathrm{BMI}+$ constant. Insulin-related variables were $\log$ or square-root transformed to normalise their distributions. Coefficient significances are: ${ }^{\mathrm{a}} p<0.05,{ }^{\mathrm{b}} p<0.01,{ }^{\mathrm{c}} p<0.001$. Borderline significances are shown in full

nation rate was a significant independent predictor of increasing MFG $(p<0.05)$.

Onset of decline in first-phase secretion. Change point regression analysis identified the following inflection points: MFG $4.97 \mathrm{mmol} / \mathrm{l}$ for the first-phase IVGTT insulin concentration response $(p=0.001)$; MFG $5.14 \mathrm{mmol} / \mathrm{l}$ for first-phase secretion by population parameter deconvolution $(p=0.103)$; and MFG $5.42 \mathrm{mmol} / \mathrm{l}$ for first-phase secretion by the combined model $(p=0.049)$. Up to these inflection points there was little change in first-phase secretion with MFG, but thereafter there was a near-linear decline. The rate of decline was calculated as 3.8, 4.2 and $4.5 \%$ per $0.1 \mathrm{mmol} / \mathrm{l}$ increase in FPG. Mean first-phase insulin output according to FPG category is illustrated for each method (Fig. 1).

Sensitivity of late-phase secretion to glucose. The sensitivity, for all three measures, of late-phase insulin release to the accompanying glucose stimulus is shown in Table 5. Coefficients for late-phase IVGTT insulin area and late-phase secretion by population parameter 
a

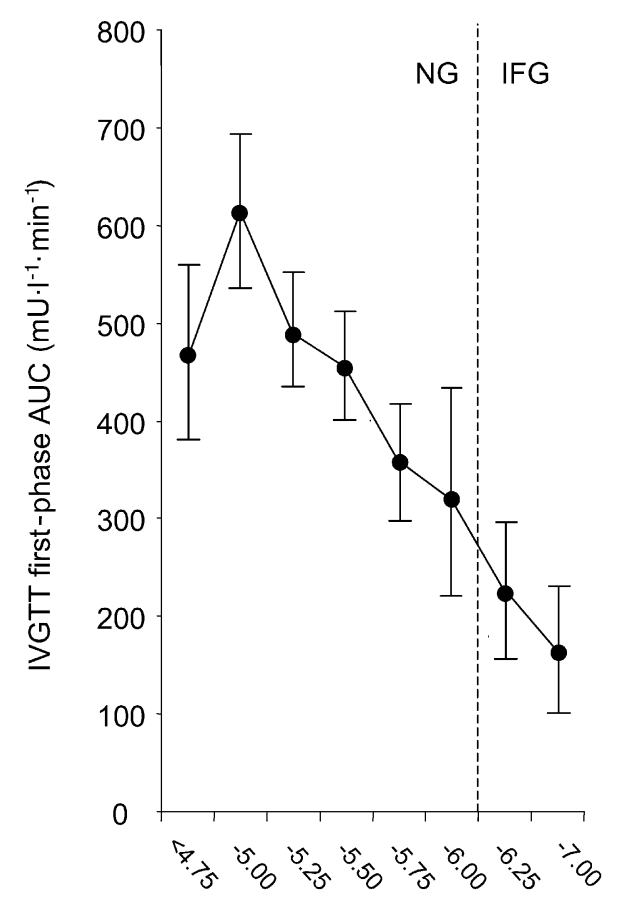

Fasting plasma glucose $(\mathrm{mmol} / \mathrm{l})$ b

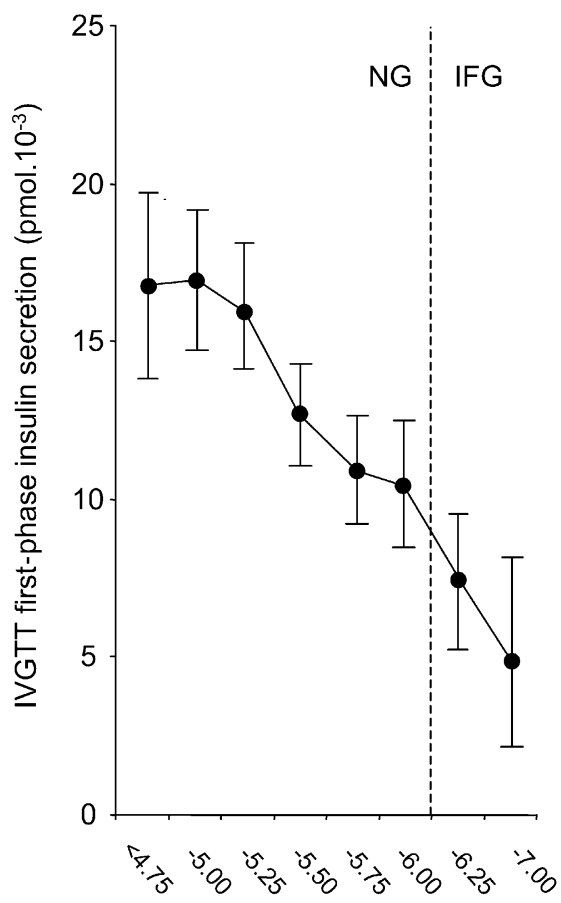

Fasting plasma glucose $(\mathrm{mmol} / \mathrm{l})$
C

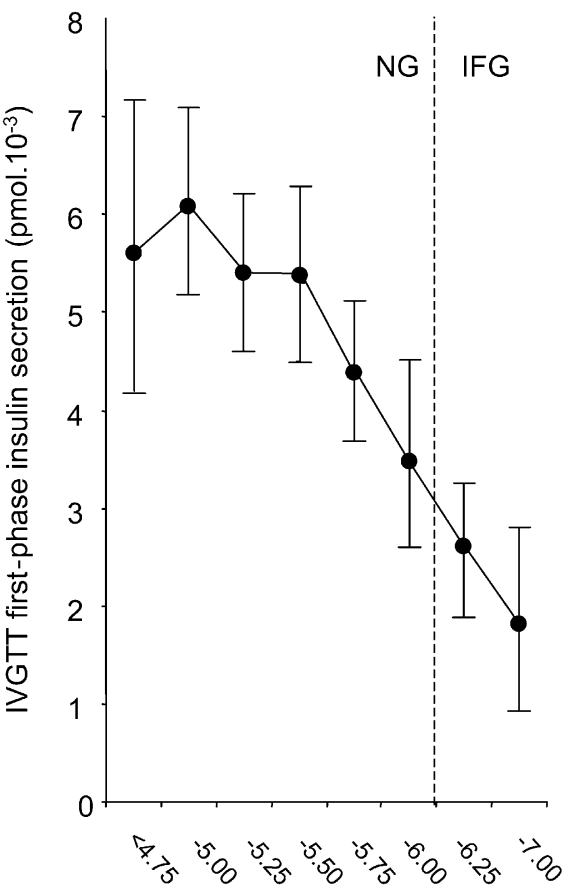

Fasting plasma glucose $(\mathrm{mmol} / \mathrm{l})$
Fig. 1. Intravenous glucose tolerance test first-phase insulin concentration and secretion responses (mean and 95\% CI) according to successively deteriorating mean fasting glucose. a. IVGTT first-phase insulin concentration area under the curve (AUC); b. net IVGTT first-phase pancreatic insulin secretion by population parameter deconvolution; c. net IVGTT first-phase pancreatic insulin secretion by the combined model of pancreatic insulin secretion. Numbers $(n)$ of subjects per fasting glucose category, see corresponding numbers in Table 2. Broken line: interface between normoglycaemia (NG; FPG $<6.1 \mathrm{mmol} / \mathrm{l}$ ) and impaired fasting glucose (IFG: 6.1-7.0 $\mathrm{mmol} / \mathrm{l}$ )

Table 5. Coefficients (95\% CI in brackets) for regression of measures of late-phase insulin release on late-phase glucose according to range of mean fasting glucose (MFG) and IVGTT k value ${ }^{a}$

Regression coefficient $\left(\times 10^{5}\right)$

Plasma insulin concentration area Pancreatic insulin secretion Pancreatic insulin secretion
(population parameter deconvolution) (combined model)

$\begin{array}{lc}\text { MFG range } & \\ <4.75 & 8.0(1.4,14.5) \\ 4.75-5.00 & 7.5(3.9,11.2) \\ 5.00-5.25 & 10.9(7.0,14.8) \\ 5.25-5.50 & 6.4(2.7,10.0) \\ 5.50-5.75 & 6.5(2.8,10.2) \\ 5.75-6.00 & 4.0(-3.2,11.3) \\ 6.00-6.25 & -3.4(-10.2,3.5) \\ 6.25-7.00 & 5.1(-2.0,12.2) \\ \text { IVGTT k value range } & \\ >2.4 & 3.6(-15.2,22.3) \\ 2.1-2.4 & 2.1(-17.5,21.8) \\ 1.8-2.1 & 15.6(3.7,27.5) \\ 1.5-1.8 & 12.6(2.9,22.3) \\ 1.2-1.5 & 10.2(4.0,16.3) \\ 1.0-1.2 & 9.2(3.4,15.1) \\ 0.8-1.0 & 17.4(11.0,23.9) \\ <0.8 & 5.0(-3.1,13.2)\end{array}$

$6.6(3.5,9.7)$
$5.0(3.0,6.9)$
$6.5(3.7,9.4)$
$6.1(3.9,8.4)$
$6.4(4.1,8.8)$
$1.7(-3.5,6.8)$
$1.6(-0.8,4.1)$
$3.3(-1.1,7.6)$

$6.1(-1.9,14.0)$

$8.7(2.9,14.4)$

$4.5(-1.0,9.9)$

$7.2(-0.3,14.7)$

$6.9(1.4,12.3)$

$-7.6(-19.1,3.9)$

$-3.3(-11.7,5.1)$

$3.0(-5.5,11.5)$
$24.6(-9.7,59.0)$

$17.7(-21.7,5.7)$

$-1.5(-21.4,18.4)$

$8.3(-7.2,23.9)$

$-5.3(-15.3,4.7)$

$8.6(-0.5,17.7)$

$6.9(-2.3,16.0)$

$3.3(-8.3,15.0)$

a For numbers in each range see Tables 2 and 3. Coefficients quantify the sensitivity of late-phase insulin release to glucose in each range of glucose homeostasis 
deconvolution were all significant (95\% confidence intervals not including zero) in categories of MFG below $6.0 \mathrm{mmol} / \mathrm{l}$. Above $6.0 \mathrm{mmol} / \mathrm{l}$, coefficients in categories of MFG for all three measures of late-phase insulin secretion (IVGTT insulin area, population parameter deconvolution and combined model) were all lower than those for categories below $6.0 \mathrm{mmol} / \mathrm{l}$ and were non-significant. Accordingly, although the 95\% confidence intervals for the coefficients in each range of MFG overlapped, there appeared to be a loss of sensitivity above MFG $6.0 \mathrm{mmol} / \mathrm{l}$. No such trends in the sensitivity of late-phase insulin response to glucose were discernable across ranges of IVGTT $k$ value.

\section{Discussion}

According to our findings, in men with non-diabetic fasting glucose levels, a marked decline in first-phase insulin release begins at levels of FPG between 5.0 and $5.4 \mathrm{mmol} / \mathrm{l}$, i.e. well within the normal range. At levels of FPG beyond 5.0 to $5.4 \mathrm{mmol} / \mathrm{l}$, first-phase beta cell function is lost at a rate of 3.8 to $4.5 \%$ per $0.1 \mathrm{mmol} / 1$ increase in FPG. These observations extend existing knowledge of the role of low first-phase insulin secretion in declining glucose homeostasis, because they define, in a large sample, the range of FPG levels at which first-phase secretion begins to deteriorate. Our findings suggest that FPG provides a highly discriminatory index of declining beta cell function, since first-phase secretion began to decline in the range of 5.0 to $5.4 \mathrm{mmol} / \mathrm{l}$ and late-phase secretion at levels above $6.0 \mathrm{mmol} / \mathrm{l}$. Moreover, no relationship was apparent between IVGTT glucose elimination and the sensitivity of late-phase insulin secretion to glucose, which is consistent with the idea that IVGTT glucose elimination is primarily determined by firstphase secretion. Our findings also demonstrate how the decline in beta cell function in the non-diabetic range of FPG occurs against a background of changes in insulin sensitivity that are primarily dependent on age and BMI. The decline in glucose effectiveness, $\mathrm{SG}$, with deteriorating glucose homeostasis is more difficult to interpret on account of the artefactual dependency of this parameter on pancreatic insulin secretion [35]. Loss of pancreatic beta cell function in the first phase is, therefore, the critical factor in declining glucose homeostasis in the non-diabetic range of FPG. A new finding of this study is that insulin elimination, both hepatic and peripheral, changes with deteriorating glucose homeostasis.

The key role of loss of first-phase insulin secretion in deteriorating glucose homeostasis has long been recognised [36, 37, 38] and the increased late-phase insulin secretion necessary to correct the initial deficit provided the original explanation for the hyperinsulinaemia seen in impaired glucose homeostasis. Subse- quently, however, studies of the pathogenesis of Type 2 diabetes have focussed on reduced insulin sensitivity, interpreting the hyperinsulinaemia primarily in terms of a compensatory response to insulin resistance [39]. Our analysis re-emphasises the importance of loss of the first-phase insulin response, independent of any decline in insulin sensitivity, and is consistent with the original observations, albeit in smaller numbers, of a previous study [19] and with a recent large cross-sectional study of Japanese volunteers, in whom the early insulin response during an OGTT was evaluated [40]. However, pancreatic insulin secretion, insulin sensitivity and insulin elimination were not measured in these studies, nor was the onset of the decline in secretion analysed.

In our study, declining glucose homeostasis was primarily defined by FPG, and some of our participants may have had impaired glucose tolerance, or even diabetic OGTT glucose levels, despite having non-diabetic FPG. The IVGTT itself has, in the past, been used to assess glucose tolerance, with an IVGTT $\mathrm{k}$ value of less than 0.8 being taken as a diagnostic criterion for diabetes. Of the 553 individuals we studied, $27 \mathrm{had} \mathrm{k}$ values in this diabetic range, but their MFG values were broadly distributed throughout the nondiabetic range (results not shown). Our study was concerned with glucose homeostasis as a continuum, represented either by FPG or IVGTT $\mathrm{k}$ value. That continuum included a variety of categories of glucose tolerance. Nevertheless, it discerned distinct relationships between measures of glucose homeostasis and insulin secretion, although it will be important to confirm the inflection in first-phase secretion with larger numbers of individuals in the categories of most effective glucose homeostasis.

The combined model of pancreatic insulin secretion involves a number of simplifying assumptions, including constant hepatic uptake of newly secreted insulin, and the new interpretations of the relationships we observed between insulin elimination and glucose homeostasis will need to be confirmed experimentally. Nevertheless, a fat feeding experiment in animals showed an inverse relationship between hepatic insulin uptake and pancreatic insulin secretion, a finding which is consistent with our observation that percent hepatic insulin extraction increases with deteriorating glucose homeostasis [41]. Increased hepatic uptake of newly secreted insulin could help counteract the impaired suppression of hepatic gluconeogenesis accompanying a deficit in first-phase pancreatic insulin output. The significant decline we observed in plasma insulin elimination rate with deteriorating glucose homeostasis could also compensate for loss of insulin secretion. However, this association was not seen with the IVGTT k value and it should be noted that, rather than specific insulin, the assay used in our study measured total insulin immunoreactivity. Insulin concentration measures therefore included 
not only insulin but insulin propeptides. Possibly, as fasting glycaemia increased, insulin propeptides with longer half-lives comprised an increasing proportion of the total insulin secreted.

Despite embodying different principles and, in two instances, using completely different measurements (insulin alone or C-peptide alone), the different measures of insulin secretion explored by us provided similar estimates of the relative impairment in firstphase secretion as FPG increased. The reference method for clinical measurement of pre-hepatic pancreatic insulin secretion is deconvolution of plasma C-peptide concentrations using rate constants and volumes of distribution derived by two-compartment modelling of the decay of plasma C-peptide concentrations after a bolus injection of synthetic C-peptide $[42,43]$. In a recent methodological comparison, both the combined model and population parameter deconvolution approaches to estimating pancreatic secretion rates provided secretion measures which were consistent with those from the reference method [44]. Comparison of secretion estimates from the two methods is nevertheless problematic, given that the population parameter approach utilises regression coefficients derived from $\mathrm{C}$-peptide concentrations measured at single centre and that combined model measurements depend on the relative accuracy of the C-peptide and insulin measurements. There may be considerable variability in these measurements. Moreover, the two methods differ in the way in which the C-peptide volume of distribution is calculated and the way in which the distribution of C-peptide is described. Equivalence in estimates of absolute secretion rates between the two methods is, therefore, not assured, and we in fact did find some differences. Nevertheless, the different secretion estimates provided for a virtually identical account of glucose homeostasis.

It is also noteworthy that the combined model was associated with a relatively high failure rate. Two contributing factors may have been: (i) the reduced sampling schedule we used; and (ii) a phenomenon we have encountered previously, whereby, in certain IVGTTs, molar concentrations of insulin and C-peptide are unexpectedly similar at the outset of the IVGTT, which tends to result in negative estimates for percent hepatic insulin extraction and therefore model failure [45]. The combined model also returned appreciably lower IVGTT secretion estimates, specifically during the late phase.

In summary, in men without diabetes, a decline in the first phase of the insulin response to glucose is a key feature of deteriorating glucose homeostasis and this commences at levels of glycaemia well within conventional normal limits. FPG levels provide a discriminatory index of the loss of beta cell function, with deterioration in first-phase response becoming apparent at FPG levels between 5.0 and $5.4 \mathrm{mmol} / \mathrm{l}$ and in late-phase insulin release at levels above
$6.0 \mathrm{mmol} / \mathrm{l}$. Novel observations, which will require further study, are an increase in the proportion of newly secreted insulin taken up by the liver with declining glucose homeostasis and a decline in the plasma insulin elimination rate with increasing fasting glycaemia.

Acknowledgements. Professor Victor Wynn initiated and established the HDDRISC study. The study was funded by the Atherosclerosis Research Trust, the Heart Disease and Diabetes Research Trust and the Cecil Rosen Foundation. I.F. Godsland and J.A.R. Jeffs were supported by the Heart Disease and Diabetes Research Trust. We extend our thanks to the many clinical, scientific, technical, nursing and administrative staff who have contributed to the study since it began, in particular Drs Carl Felton, Anthony Proudler, Christopher Walton, Raymond Bruce, Francisco Leyva and John Stevenson. We thank Professor Richard Bergman and Dr Richard Watanabe for providing the programs for analyses using the combined and the extended combined models of pancreatic insulin secretion, and Professor Kenneth Polonsky for providing the program for deconvolution analysis.

\section{References}

1. Gerich JE (1998) The genetic basis of Type 2 diabetes mellitus: impaired insulin secretion versus impaired insulin sensitivity. Endocrine Rev 19:491-503

2. Pratley RE, Weyer C (2001) The role of impaired early insulin secretion in the pathogenesis of type II diabetes mellitus. Diabetologia 44:929-945

3. Straub SG, Sharp GWG (2002) Glucose-stimulated signaling pathways in biphasic insulin secretion. Diabetes Metab Res Rev 18:451-463

4. Lillioja S, Mott DM, Spraul M et al. (1993) Insulin resistance and insulin secretory dysfunction as precursors of non-insulin-dependent diabetes mellitus. Prospective studies of Pima Indians. N Engl J Med 329:1988-1992

5. Haffner SM, Miettinen H, Gaskill SP, Stern MP (1995) Decreased insulin secretion and increased insulin resistance are independently related to the 7-year risk of NIDDM in Mexican-Americans. Diabetes 44:1386-1391

6. Gerich JE (2002) Is reduced first-phase insulin release the earliest detectable abnormality in individuals destined to develop type 2 diabetes. Diabetes 51 [Suppl 1]:S117-S121

7. Zethelius B, Byberg L, Hales CN, Lithell H, Berne C (2003) Proinsulin and acute insulin response independently predict type 2 diabetes mellitus in men-report from 27 years of follow-up study. Diabetologia 46:20-26

8. Pimenta W, Korytkowski M, Mitrakou A et al. (1995) Pancreatic beta-cell dysfunction as the primary genetic lesion in NIDDM. Evidence from studies in normal glucose-tolerant individuals with first-degree NIDDM relative. JAMA 273:1855-1861

9. Vølund A, Polonsky K, Bergman R (1987) Calculated pattern of intraportal insulin appearance without independent assessment of C-peptide kinetics. Diabetes 36:1195-1202

10. Cobelli C, Pacini G (1988) Insulin secretion and hepatic extraction in humans by minimal modelling of C-peptide and insulin kinetics. Diabetes 37:223-231

11. Ferrannini E, Cobelli C (1987) The kinetics of insulin in man. II. Role of the liver. Diabetes Metab Rev 3:365-397

12. Gower BA, Granger WM, Franklin F, Shewchuk RM, Goran MI (2002) Contribution of insulin secretion and clearance to glucose-induced insulin concentration in African-American and Caucasian children. J Clin Endocrinol Metab 87:18-24 
13. Goran MI, Bergman RN, Cruz ML, Watanabe R (2002) Insulin resistance and associated compensatory responses in African-American and Hispanic children. Diabetes Care 25:2184-2190

14. The Expert Committee on the Diagnosis and Classification of Diabetes Mellitus. (1997) Report of the Expert Committee on the diagnosis and classification of diabetes mellitus. Diabetes Care 20:1183-1197

15. Alberti KGM, Zimmet PZ, for the WHO Consultation (1998) Definition, diagnosis and classification of diabetes mellitus and its complications. Part 1: Diagnosis and classification of diabetes mellitus. Provisional Report of a WHO Consultation. Diabetic Med 15:539-553

16. Charles MA, Fontbonne A, Thibult N, Warnet J-M, Rosselin GE, Eschwege E (1991) Risk factors for NIDDM in white population: Paris Prospective Study. Diabetes 40:796-799

17. Coutinho M, Gerstein HC, Wang Y, Yusuf S (1999) The relationship between glucose and incident cardiovascular events. Diabetes Care 22:233-240

18. Shaw JE, Zimmet PZ, Hodge AM et al. (2000) Impaired fasting glucose: how low should it go? Diabetes Care 23:34-39

19. Brunzell JD, Robertson RP, Lerner RL et al. (1976) Relationship between fasting plasma glucose levels and insulin secretion during intravenous glucose tolerance. J Clin Endocrinol Metab 42:222-229

20. Van Haeften TW, Pimenta W, Mitrakou A et al. (2000) Relative contributions of beta-cell function and tissue insulin sensitivity to fasting and postglucose-load glycaemia. Metabolism 49:1318-1325

21. Godsland IF, Leyva F, Worthington M, Walton C, Stevenson JC (1998) Associations of smoking, alcohol and physical activity with risk factors for coronary heart disease and diabetes in the first follow-up cohort of the HDDRISC Study (HDDRISC-1). J Intern Med 244:33-41

22. Godsland IF, Rosankiewicz JR, Proudler AJ, Johnston DG (2001) Plasma total homocysteine concentrations are unrelated to insulin sensitivity and components of the metabolic syndrome in healthy men. J Clin Endocrinol Metab 86:719-723

23. Trinder P (1969) Determination of blood glucose using an oxidase-peroxidase system with non-carcinogenic chromogen. J Clin Path 22:158-161

24. Albano JDM, Ekins RP, Maritz G, Turner RC (1972) A sensitive, precise radioimmunoassay of serum insulin relying on charcoal separation of bound and free hormone moieties. Acta Endocrinol 70:487-509

25. Bergman RN, Ider YZ, Bowden CR, Cobelli C (1979) Quantitative estimation of insulin sensitivity. Am J Physiol 236:E667-E677

26. Bergman R (1989) Lilly Lecture 1989. Towards physiological understanding of glucose tolerance: minimal model approach. Diabetes 38:1512-1527

27. Walton C, Godsland IF, Proudler AJ, Felton C, Wynn V (1992) Evaluation of four mathematical models of glucose and insulin dynamics with analysis of the effects of age and obesity. Am J Physiol 262:E755-E762

28. Swan J, Walton C, Godsland IF (1994) Assessment of insulin sensitivity in man: a comparison of minimal model and euglycaemic clamp derived measures in health and heart failure. Clin Sci 86:317-322
29. Horwitz D, Starr J, Mako M, Blackard W, Rubenstein A (1975) Proinsulin, insulin and C-peptide concentrations in human portal and peripheral blood. J Clin Invest 55:1278-1283

30. Van Cauter E, Mestrez F, Sturis J, Polonsky K (1992) Estimation of insulin secretion rates from C-peptide levels. Comparison of individual and standard kinetic parameters for C-peptide clearance. Diabetes 41:368-377

31. Watanabe R, Vølund A, Roy S, Bergman R (1989) Prehepatic beta-cell secretion during the intravenous glucose tolerance test in humans: Application of a combined model of insulin and C-peptide kinetics. J Clin Endocrinol Metab 69:790-797

32. Tranberg K, Dencker H (1978) Modeling of plasma disappearance of unlabelled insulin in man. Am $\mathrm{J}$ Physiol 235:E577-E585

33. Watanabe RM, Steil GM, Bergman RN (1998) Critical evaluation of the combined model approach for estimation of prehepatic insulin secretion. Am J Physiol 274:E172E183

34. Julious SA (2001) Inference and estimation in changepoint regression. J Royal Stat Soc 50:51-62

35. Finegood DT, Tzur D (1996) Reduced glucose effectiveness associated with reduced insulin release: an artifact of the minimal-model method. Am J Physiol 271:E485-E495

36. Seltzer H, Allen E, Herron A Jr, Brennan M (1967) Insulin secretion in response to glycemic stimulus: relation of delayed initial release to carbohydrate intolerance in mild diabetes mellitus. J Clin Invest 46:323-335

37. Perley M, Kipinis D (1967) Plasma insulin responses to oral and intravenous glucose: studies in normal and diabetic subjects. J Clin Invest 46:1954-1962

38. Cerasi E (1967) An analogue computer model for the insulin response to glucose infusion. Acta Endocrinol 55:163-183

39. DeFronzo RA (1988) Lilly lecture 1987: the triumvirate: beta cell, muscle, liver: a collusion responsible for NIDDM. Diabetes 37:667-687

40. Ozaki K, Okubo M, Mori H, Mito K, Hara H, Kohno N (2002) Decreased insulin secretion and dyslipidemia coexist in subjects with impaired fasting glucose. Diabetes Res Clin Pract 55:159-164

41. Mittelman SD, van Citters GW, Kim SP et al. (2000) Longitudinal compensation for fat-induced insulin resistance includes reduced insulin clearance and enhanced beta-cell response. Diabetes 49:2116-2125

42. Eaton R, Allen R, Schade D, Erickson K, Standefer J (1980) Prehepatic insulin production in man: kinetic analysis using peripheral connecting peptide behaviour. J Clin Endocrinol Metab 51:520-528

43. Polonsky K, Licinio-Paixao J, Given B et al. (1986) Use of biosynthetic human $\mathrm{C}$-peptide in the measurement of insulin secretion rates in normal volunteers and type 1 diabetic patients. J Clin Invest 77:98-105

44. Kjems LL, Volund A, Madsbad S (2001) Quantification of beta-cell function during IVGTT in type II and non-diabetic subjects: assessment of insulin secretion by mathematical methods. Diabetologia 44:1339-1348

45. Walton C, Godsland I, Proudler A, Felton C, Wynn V (1992) An apparently anomalous relationship between insulin and C-peptide concentrations in their initial response to intravenous glucose. Metabolism 41:1210-1214 